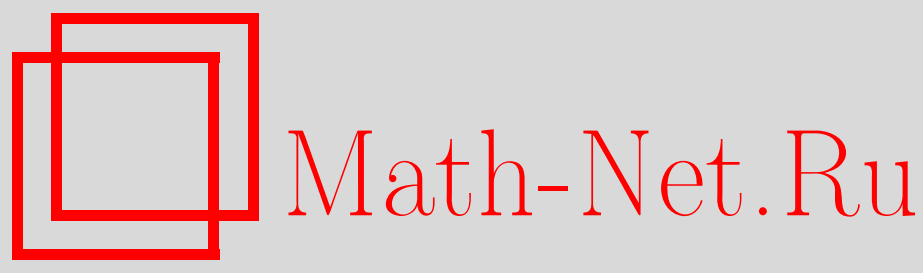

С. В. Востоков, И. Л. Климовицкий, Примарные элементы в формальных модулях, Совр. пробл. матем., 2013, выпуск 17, 153-163

DOI: https://doi.org/10.4213/spm48

Использование Общероссийского математического портала Math-Net.Ru подразумевает, что вы прочитали и согласны с пользовательским соглашением http://www.mathnet.ru/rus/agreement

Параметры загрузки:

IP : 54.172 .240 .79

26 апреля 2023 г., 12:50:33

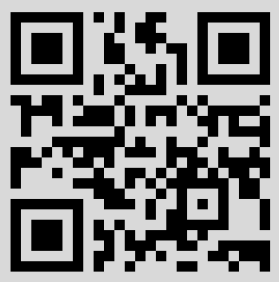




\title{
Примарные элементы в формальных модулях
}

\author{
С. В. Востоков, И. Л. Климовицкий \\ Санкт-Петербургский государственный университет
}

Замечательному ученому и человеку посвящается

В арифметике формальных модулей, построенных на идеалах полных дискретно нормированных полей с помощью формальных групповых законов, существенную роль играют так называемые примарные элементы, которые дают неразветвленные расширения. В настоящей работе мы получаем примарные элементы в произвольном формальном модуле локального поля с совершенным полем вычетов. При этом в качестве формальных групповых законов мы рассматриваем так называемые формальные $O_{0}$-модульные группы.

В разделе 1 строятся примарные элементы типа Хассе, в определение которых входит элемент из кольца целых пополнения максимального неразветвленного расширения данного поля.

В разделе 2 конструируются примарные элементы, аналогичные тем, которые впервые были найдены в работах [1], [2] и в определение которых входят лишь элементы исходного поля. Для этого излагается теория Хонды для формальных $O_{0}$-модулей, а затем на основании этой теории доказывается теорема о представлении логарифма формального $O_{0}$-модуля в виде произведения двух множителей. Далее строятся функции Артина-Хассе для универсальных формальных $O_{0}$-модулей и, наконец, доказываются основные теоремы.

\section{1. Формальные модули}

1.1. Формальные $\boldsymbol{O}_{0}$-модульные группы. Пусть $K_{0}$ - локальное поле (конечное расширение $\left.\mathbb{Q}_{p}\right)$ с кольцом целых $O_{0}$ и простым элементом $\pi_{0}$. Пусть, далее, $K$ - конечное расширение поля $K_{0}, O:=O_{K}$ - кольцо целых поля $K$ и $\pi$ - простой элемент в $K$.

ОПРЕДЕЛЕНИЕ 1. Формальную группу $F(X, Y)$ над кольцом $O$ с логарифмом $\lambda(X) \in$ $K \llbracket X \rrbracket$ назовем формалъной $O_{0}$-модульной группой, если определен кольцевой гомоморфизм

$$
\begin{aligned}
{[\cdot]_{F}: O_{0} } & \rightarrow \operatorname{End}_{O}(F), \\
a & \mapsto[a]_{F}(X)=\lambda^{-1}(a \lambda(X)) .
\end{aligned}
$$

Заметим, что если $f: F \rightarrow G$ - гомоморфизм формальных $O_{0}$-модульных групп $F$ и $G$ над $O$, то

$$
f(X) \circ[a]_{F}(X)=[a]_{G}(X) \circ f(X)
$$

(подробнее см. [3; гл. IV, §27]).

1.2. Высота формальной $\boldsymbol{O}_{0}$-модульной группы. Пусть $F(X, Y)$ - формальная $O_{0}$-модульная группа над $O$ высоты $h$ и $m=\left(K_{0}: \mathbb{Q}_{p}\right)$.

ОПРЕДЕЛЕНИЕ 2. Формалъной $O_{0}$-высотой группы $F$ над $O$ назовем число

$$
h_{0}=\frac{h}{m} .
$$

Работа выполнена при финансовой поддержке Российского фонда фундаментальных исследований (грант № 12-01-33057).

(c) С. В. Востоков, И. Л. Климовицкий, 2013 
Можно проверить, что $h_{0}$ является натуральным числом или бесконечностью. При этом $h_{0}=\infty$ тогда и только тогда, когда

$$
\pi_{0}^{e} \lambda(X) \equiv 0 \quad \bmod \pi_{0} O
$$

где $e=e\left(K / K_{0}\right)($ см. [3]).

1.3. Функция Артина-Хассе формальной $\boldsymbol{O}_{0}$-модульной группы. Пусть $F(X, Y)$ формальная $O_{0}$-модульная группа над $O$ конечной формальной $O_{0}$-высоты $h_{0}$. Пусть $L-$ некоторое полное дискретно нормированное поле с совершенным полем вычетов $\bar{L}$. Считаем, что $L$ содержит $K$ и, тем самым, $K_{0}$. Пусть $T$ - максимальное абсолютно неразветвленное над $K_{0}$ подполе в $L / K_{0}$ с кольцом целых $O_{T}$. Пусть $\widehat{T^{n r}}-$ пополнение максимального неразветвленного расширения поля $T, \varphi$ - автоморфизм Фробениуса в $\widetilde{T^{n r}} / K_{0}$; таким образом,

$$
\varphi(\alpha)=\alpha^{\varphi} \equiv \alpha^{q} \quad \bmod \pi_{0}
$$

для всех $\alpha \in O_{\widetilde{T^{n r}}}$ (здесь $q$ - число элементов поля вычетов $\overline{K_{0}}$ ).

Пусть $\mathfrak{M}:=\mathfrak{M}_{L}-$ максимальный идеал кольца целых поля $L$ и $F(\mathfrak{M})$ - формальный $O_{0}$-модуль, натянутый на идеал $\mathfrak{M}$ :

$$
\begin{aligned}
\alpha, \beta \in F(\mathfrak{M}) & \mapsto \alpha+{ }_{F} \beta=F(\alpha, \beta) \in F(\mathfrak{M}), \\
a \in O_{0}, \alpha \in F(\mathfrak{M}) & \mapsto[a]_{F}(\alpha) \in F(\mathfrak{M}) .
\end{aligned}
$$

Пусть $\lambda(X)$ - логарифм $F$ и $\lambda_{q}(X)$ - логарифм $O_{0}$-типической формальной группы $F_{q}$, изоморфной $F$. Заметим, что $F_{q}$ определена не однозначно. Например, в качестве $\lambda_{q}(X)$ можно брать ряд

$$
\lambda_{q}(X)=X+c_{q} X^{q}+c_{q^{2}} X^{q^{2}}+\cdots,
$$

где

$$
\lambda(X)=X+c_{1} X+c_{2} X^{2}+\cdots,
$$

или ряд $\lambda_{q}(X)=v(\Delta) u(\Delta)^{-1}(X)$ из теории Хонды. Тогда ряд $\left(\lambda^{-1} \circ \lambda_{q}\right)(X) \in O \llbracket X \rrbracket$ и задает изоморфизм из $F$ в $F_{q}$ (см. [3]).

ОПРЕДЕЛЕНИЕ 3. Для любого элемента $\alpha \in O_{\widetilde{T}^{n r}}$ определим ряд, который назовем $\oint y н к-$ иией Артина-Хассе:

$$
E_{F}(\alpha X)=\lambda^{-1}\left(\alpha X+c_{q} \varphi(\alpha) X^{q}+c_{q^{2}} \varphi^{2}(\alpha) X^{q^{2}}+\cdots\right)
$$

(в мультипликативном случае ряд $E(X)$ определен в работе [4]).

ПреДЛОЖЕНИЕ 1. Для любого элемента $\mathfrak{Z} \in F(\mathfrak{M})$ элемент $E_{F}(\alpha \mathfrak{Z})$ корректно определен и задает элемент из $F(\widetilde{\mathfrak{M}})$, где $\widetilde{\mathfrak{M}}$ - максимальный идеал в кольце цельх поля $L_{T^{n r}}$. При этом

$$
\begin{aligned}
E_{F}((\alpha+\beta) \mathfrak{Z}) & =E_{F}(\alpha \mathfrak{Z})+{ }_{F} E_{F}(\beta \mathfrak{Z}), & \alpha, \beta & \in O_{\widetilde{T}^{n r}}, \\
E_{F}(a \alpha \mathfrak{Z}) & =[a]_{F}\left(E_{F}(\alpha \mathfrak{Z})\right), & a & \in O_{0}, \alpha \in O_{\widetilde{T}^{n r}}, \\
E_{F}(\alpha \mathfrak{Z}) & \equiv \alpha \mathfrak{Z} \bmod \alpha \mathfrak{Z}^{2}, & \alpha & \in O_{\widetilde{T}^{n r}} .
\end{aligned}
$$

ДокАЗАТЕльство. 1) Пусть $R$ - мультипликативная система представителей Тейхмюллера в $\widetilde{T^{n r}}$. Тогда элемент $\alpha \in O_{\widetilde{T}^{n r}}$ представи́м в виде

$$
\alpha=\theta_{0}+\theta_{1} \pi_{0}+\theta_{2} \pi_{0}^{2}+\cdots=\sum_{i \geqslant 0} \theta_{i} \pi_{0}^{i}, \quad \theta_{i} \in R,
$$


при этом $\varphi\left(\theta_{i}\right)=\theta_{i}^{q}$, где $q=\# \overline{K_{0}}$ - число элементов поля вычетов $\overline{K_{0}}$ поля $K_{0}$. Поэтому

$$
\begin{aligned}
E_{F}(\alpha \mathfrak{Z}) & =\lambda^{-1}\left(\sum_{i \geqslant 0} c_{q^{i}}\left(\theta_{0} \mathfrak{Z}\right)^{q^{i}}+\pi_{0} \sum_{i \geqslant 0} c_{q^{i}}\left(\theta_{1} \mathfrak{Z}\right)^{q^{i}}+\cdots\right) \\
& =\lambda^{-1}\left(\sum_{i \geqslant 0} c_{q^{i}}\left(\theta_{0} \mathfrak{Z}\right)^{q^{i}}\right)+{ }_{F}\left[\pi_{0}\right]_{F}\left(\lambda^{-1}\left(\sum_{i \geqslant 0} c_{q^{i}}\left(\theta_{1} \mathfrak{Z}\right)^{q^{i}}\right)\right)+{ }_{F} \cdots \\
& =\sum_{k \geqslant 0}(F)\left[\pi_{0}^{k}\right]_{F}\left(E_{F}\left(\theta_{k} \mathfrak{Z}\right)\right) \in F(\widetilde{\mathfrak{M}}) .
\end{aligned}
$$

2) Если $\alpha=\sum_{i \geqslant 0} \theta_{i} \pi_{0}^{i}, \beta=\sum_{i \geqslant 0} \theta_{i}^{\prime} \pi_{0}^{i}$, где $\theta_{i}, \theta_{i}^{\prime} \in R$, то из предыдущего равенства в п. 1$)$ имеем

$$
E_{F}((\alpha+\beta) \mathfrak{Z})=\sum_{k \geqslant 0}(F)\left[\pi_{0}^{k}\right]_{F}\left(E_{F}\left(\left(\theta_{k}+\theta_{k}^{\prime}\right) \mathfrak{Z}\right)\right) .
$$

С другой стороны,

$$
\begin{aligned}
& E_{F}(\alpha \mathfrak{Z})+{ }_{F} E_{F}(\beta \mathfrak{Z})=\sum_{k \geqslant 0}(F)\left[\pi_{0}^{k}\right]_{F}\left(E_{F}\left(\theta_{k} \mathfrak{Z}\right)\right)+{ }_{F} \sum_{k \geqslant 0}(F)\left[\pi_{0}^{k}\right]_{F}\left(E_{F}\left(\theta_{k}{ }^{\prime} \mathfrak{Z}\right)\right) \\
& =\sum_{k \geqslant 0}(F)\left[\pi_{0}^{k}\right]_{F}\left(E_{F}\left(\theta_{k} \mathfrak{Z}+\theta_{k}^{\prime} \mathfrak{Z}\right)\right)=E_{F}((\alpha+\beta) \mathfrak{Z}) .
\end{aligned}
$$

3) Следует из того, что $\varphi(a)=a$, для $a \in O_{0}$.

4) Вытекает непосредственно из определения функции

$$
E_{F}(X)=\left(\lambda^{-1} \circ \lambda_{q}\right)(X) \equiv X \quad \bmod X^{2} .
$$

1.4. Примарные элементы в формальном $\boldsymbol{O}_{0}$-модуле $\boldsymbol{F}(\mathfrak{M})$. Примем все обозначения п. 1.3. Предположим, что поле $L$ содержит ядро изогении $\left[\pi_{0}^{n}\right]_{F}$. Назовем элемент $\omega \in$ $F(\mathfrak{M}) \pi_{0}^{n}$-примарным, если расширение $L\left(\frac{1}{\pi_{0}^{n}} \omega\right) / L$ неразветвленное, где $\frac{1}{\pi_{0}^{n}} \omega$ обозначает решение уравнения $\left[\pi_{0}^{n}\right]_{F}(X)=\omega$. Ясно, что из условия $\operatorname{ker}\left[\pi_{0}^{n}\right]_{F} \subset L$ следует, что расширение $L\left(\frac{1}{\pi_{0}^{n}} \omega\right) / L$ абелево. Пусть $\mathfrak{Z}$ - некоторый первообразный элемент из ядра $\operatorname{ker}\left[\pi_{0}^{n}\right]_{F}$, т.е. $\left[\pi_{0}^{n}\right]_{F}(\mathfrak{Z})=0$ и $\left[\pi_{0}^{n-1}\right]_{F}(\mathfrak{Z}) \neq 0$. Элемент $\mathfrak{Z} \in F(\mathfrak{M})$, и из определения функции $E_{F}$ следует, что существует такой элемент $\mathfrak{E} \in F(\mathfrak{M})$, что $\mathfrak{Z}=E_{F}(\mathfrak{E})=\left(\lambda^{-1} \circ \lambda_{q}\right)(\mathfrak{E})$.

Для любого элемента $\alpha \in O_{T}$ найдется элемент $A$ из кольца $O_{\widetilde{T}^{n r}}$ такой, что

$$
A^{\varphi}-A=\alpha .
$$

Пополнение расширения $T(A) / T$ является, согласно теории Витта, пополнением $Г$-расширения, и если $\sigma$ - образующий элемент группы Галуа этого Г-расширения, то

$$
A^{\sigma}-A=\varkappa \in O_{0} .
$$

Здесь и далее продолжение $\sigma$ на $\widetilde{T^{n r}}$ обозначим той же буквой $\sigma$.

ОПРЕДЕЛЕНИЕ 4. Определим $H_{\mathfrak{Z}}(\alpha)=E_{F}\left(\left(\pi_{0}^{n} A\right) \mathfrak{E}\right)$.

Теорема 1. 1. Элемент $H_{\mathfrak{Z}}(\alpha)$ является $\pi_{0}{ }^{n}$-примарным в бормальном $O_{0}$-модуле $F(\mathfrak{M})$, m.e.

a) $H_{\mathfrak{Z}}(\alpha) \in L$

b) расширение $L\left(\frac{1}{\pi_{0}{ }^{n}} H_{\mathfrak{Z}}(\alpha)\right) / L$ является конечным абелевым неразветвленным расширением.

2. Примарный элемент $H_{\mathfrak{Z}}(\alpha)$ не зависит от выбора элемента $A$ из уравнения $A^{\varphi}-A=\alpha$. 
ДокАЗАТЕльство. Для доказательства п. 1 нам понадобится следующая

ЛЕмма 1. Пусть $x \in \widetilde{T(A)}$ u $\sigma(x)=x ;$ тогда $x \in T$, где $\widetilde{T(A)}-$ пополнение $T(A)$.

ДокАЗАТЕЛЬСтво. Пусть $T_{\Gamma} / T$ - алгебраическая часть расширения $\widetilde{T(A)} / T$. Как уже было отмечено выше, это Г-расширение. Теперь пусть $\theta$ - некоторый представитель Тейхмюллера в $\widetilde{T(A)}$. Тогда ясно, что $\theta$ также будет представителем Тейхмюллера в конечном расширении поля $T$ (с полем вычетов $\bar{T}(\bar{\theta})$ ), которое полно. Таким образом, $\theta$ - алгебраический элемент над $T$, лежащий в $T_{\Gamma} / T$.

Рассмотрим разложение

$$
x=\theta_{0}+\theta_{1} \pi_{0}+\theta_{2} \pi_{0}^{2}+\cdots .
$$

Тогда

$$
\sigma(x)=\sigma\left(\theta_{0}\right)+\sigma\left(\theta_{1}\right) \pi_{0}+\sigma\left(\theta_{2}\right) \pi_{0}^{2}+\cdots .
$$

Заметим, что под действием автоморфизма представитель Тейхмюллера перейдет в элемент, из которого также извлекаются все корни $p^{n}$ степени, т.е. в представитель Тейхмюллера. Таким образом,

$$
\sigma(x)=\theta_{0}^{\prime}+\theta_{1}^{\prime} \pi_{0}+\theta_{2}^{\prime} \pi_{0}^{2}+\cdots .
$$

Если $x=\sigma(x)$, то $\theta_{i}=\theta_{i}^{\prime}=\sigma\left(\theta_{i}\right)$ для всех $i \geqslant 0$. Но тогда $\theta_{i} \in T$ (ведь $\sigma$ - топологическая образующая $\left.\operatorname{Gal}\left(T_{\Gamma} / T\right)\right)$. Таким образом, $x$ лежит в $T$.

Ясно, что $\mathfrak{E}$ является корнем изогении $\left[\pi_{0}^{n}\right]_{F_{q}}$ (она имеет конечную высоту, равную высоте изогении $\left.\left[\pi_{0}^{n}\right]_{F}\right)$, лежащим в $F(\mathfrak{M})$; таким образом, $\mathfrak{E}$ является алгебраическим над $K$.

По построению $H_{\mathcal{3}}(\alpha)$ лежит в $\left.\widetilde{K(\mathfrak{E}, A}\right)$ (как ряд от $\left.\mathfrak{E}, A, A^{\varphi}, A^{\varphi^{2}}, \ldots\right)$ и, тем самым, в пополнении композита $K T(\mathfrak{E}, A)$. Заметим, что $K / K_{0}$ конечно и $K_{0} \subset T$; следовательно, $K T(\mathfrak{E}) / T$ конечно. Пусть тогда $K T(\mathfrak{E})=T\left(\mathfrak{E}^{\prime}\right)$.

Заметим теперь, что $T\left(\mathfrak{E}^{\prime}\right) / T$ - вполне разветвленное расширение, тогда как $\widetilde{T(A)} / T-$ неразветвленное. Таким образом, их пересечением является в точности $T$. И мы получаем, что в расширении $T\left(A, \mathfrak{E}^{\prime}\right) / T\left(\mathfrak{E}^{\prime}\right)$ также действует образующий автоморфизм $\sigma$. И для того, чтобы $H_{\mathfrak{Z}}(\alpha)$ лежал в $L$ (и даже в $\left.T\left(\mathfrak{E}^{\prime}\right)\right)$, достаточно согласно лемме, чтобы $H_{\mathfrak{Z}}(\alpha)^{\sigma}=H_{\mathfrak{Z}}(\alpha)$.

Действительно,

$$
\begin{aligned}
H_{\mathfrak{Z}}(\alpha)^{\sigma} & =E_{F}\left(\pi_{0}^{n} A^{\sigma} \mathfrak{E}\right)=E_{F}\left(\pi_{0}^{n} A \mathfrak{E}\right)+{ }_{F} E_{F}\left(\pi_{0}^{n} \varkappa \mathfrak{E}\right) \\
& =H_{\mathfrak{Z}}(\alpha)+{ }_{F}\left[\pi_{0}^{n} \varkappa\right]_{F}\left(E_{F}(\mathfrak{E})\right)=H_{\mathfrak{Z}}(\alpha)+{ }_{F}[\varkappa]_{F}\left(\left[\pi_{0}^{n}\right]_{F}(\mathfrak{Z})\right)=H_{\mathfrak{Z}}(\alpha) .
\end{aligned}
$$

Для первого равенства нам необходима коммутируемость $\sigma$ и $\varphi$ на $A$. Это так в силу того, что $A^{\sigma \varphi}=A+a+\varkappa=A^{\varphi \sigma}$.

Покажем теперь, что расширение

$$
L\left(\frac{1}{\pi_{0}^{n}} H_{\mathfrak{Z}}(\alpha)\right) / L
$$

неразветвленное.

Пусть $t=\frac{1}{\pi_{0}^{n}} H_{\mathfrak{Z}}(\alpha)=E_{F}(A \mathfrak{E})$. Из свойств функции $E_{F}$ следует, что $t$ лежит в $F(\widetilde{\mathfrak{M}})$. Но при этом $t$ является корнем уравнения $\left[\pi_{0}^{n}\right]_{F}(X)-H_{\mathfrak{Z}}(\alpha)=0$. Ряд $\left[\pi_{0}^{n}\right]_{F}$ имеет конечную высоту в любом расширении $K$, в частности в $K T(\mathfrak{E})$. Элемент $H_{\mathfrak{Z}}(\alpha)$, как было установлено выше, лежит в поле $K T(\mathfrak{E})$ и при этом имеет в нем положительное нормирование (так как является значением функции $\left.E_{F}\right)$. Таким образом, ряд $\left[\pi_{0}^{n}\right]_{F}(X)-H_{\mathfrak{Z}}(\alpha)$ имеет конечную высоту над полем $K T(\mathfrak{E})$. И, следовательно, $t$ как его корень с положительным нормированием является алгебраическим над $K T(\mathfrak{E})$ (согласно подготовительной лемме Вейерштрасса). 
Покажем для начала, что расширение

$$
K T(A, \mathfrak{E}) / K T(\mathfrak{E})
$$

неразветвленное. Пусть, как и раньше $K T(\mathfrak{E})=T\left(\mathfrak{E}^{\prime}\right)$. Элемент $\pi_{0}-$ простой в $T$, а $\pi^{\prime}-$ простой в $T\left(\mathfrak{E}^{\prime}\right)$. Так как расшерение $T\left(\mathfrak{E}^{\prime}\right) / T$ вполне разветвлено, то

$$
e=e\left(T\left(\mathfrak{E}^{\prime}\right) / T\right)=\operatorname{deg}\left(T\left(\mathfrak{E}^{\prime}\right) / T\right) .
$$

Таким образом, $\pi^{\prime e} / \pi$ - единица в $T\left(\mathfrak{E}^{\prime}\right)$. Но тогда это отношение является также единицей в $T\left(A, \mathfrak{E}^{\prime}\right)$. Теперь рассмотрим $\pi$ как простой элемент в $T(A)$. Мы получили, что в $T\left(A, \mathfrak{E}^{\prime}\right)$ нашелся элемент $\pi^{\prime}$ такой, что $\pi^{\prime e} / \pi$ - единица. Таким образом,

$$
e\left(T\left(A, \mathfrak{E}^{\prime}\right) / T(A)\right) \geqslant e .
$$

Но очевидно, что

$$
\operatorname{deg}\left(T\left(A, \mathfrak{E}^{\prime}\right) / T(A)\right) \leqslant \operatorname{deg}\left(T\left(\mathfrak{E}^{\prime}\right) / T\right)
$$

Итак, мы получили

$$
e\left(T\left(A, \mathfrak{E}^{\prime}\right) / T(A)\right) \geqslant e=\operatorname{deg}\left(T\left(\mathfrak{E}^{\prime}\right) / T\right) \geqslant \operatorname{deg}\left(T\left(A, \mathfrak{E}^{\prime}\right) / T(A)\right) \geqslant e\left(T\left(A, \mathfrak{E}^{\prime}\right) / T(A)\right) .
$$

Это возможно тогда и только тогда, когда все неравенства обращаются в равенства. В частности, $e\left(T\left(A, \mathfrak{E}^{\prime}\right) / T(A)\right)=e$, из чего следует, что $\pi^{\prime}$ является простым элементом в $T\left(A, \mathfrak{E}^{\prime}\right)$. Таким образом, расширение $T\left(A, \mathfrak{E}^{\prime}\right) / T\left(\mathfrak{E}^{\prime}\right)$ неразветвленное. Соответственно $K T(A, \mathfrak{E}) / K T(\mathfrak{E})$ неразветвленное.

Из доказанного непосредственно следует, что $K \widetilde{T(A, \mathfrak{E})} / K T(\mathfrak{E})$ неразветвленное. По построению функции $E_{F}$ элемент $t=E_{F}(A \mathfrak{E})$ лежит в поле $K \widehat{T(A, \mathfrak{E})}$. Таким образом, расширение $K T(\mathfrak{E}, t) / K T(\mathfrak{E})$ является подрасширением $\widehat{K T(A, \mathfrak{E})} / K T(\mathfrak{E})$ и, тем самым, тоже неразветвленное.

Пусть $N=K T(\mathfrak{E})$. Выше мы доказали, что $N(t) / N$ неразветвленное. Пусть $f$ - степень инерции этого расширения (тогда $\operatorname{deg}(N(t) / N)=f$ ). Ясно, что поле $L(t)$ содержит поле $N(t)$ и, тем самым, $\overline{N(t)} \subset \overline{L(t)}$. В силу того, что $N$ содержит $T$, расширение $L / N$ вполне разветвлено и $\bar{L}=\bar{N}$. Итак,

$$
\operatorname{deg}(\overline{L(t)} / \bar{L}) \geqslant f
$$

Осталось заметить, что

$$
\operatorname{deg}(N(t) / N) \geqslant \operatorname{deg}(L(t) / L)
$$

и мы получаем

$$
f=\operatorname{deg}(N(t) / N) \geqslant \operatorname{deg}(L(t) / L) \geqslant \operatorname{deg}(\overline{L(t)} / \bar{L}) \geqslant f .
$$

Это возможно тогда и только тогда, когда все неравенства обращаются в равенства. В частности, $\operatorname{deg}(L(t) / L)=\operatorname{deg}(\overline{L(t)} / \bar{L})$, что означает неразветвленность расширения $L(t) / L$, т.е. неразветвленность $L\left(\frac{1}{\pi_{0}^{n}} H_{\mathfrak{Z}}(\alpha)\right) / L$.

Заметим, что утверждения теоремы остаются верны, какой бы из элементов $\frac{1}{\pi_{0}^{n}} H_{\mathfrak{Z}}(\alpha)$ ни был взят. Действительно, очевидно, что любой элемент вида $\frac{1}{\pi_{0}^{n}} H_{\mathfrak{Z}}(\alpha)$ представляется как

$$
E_{F}(A \mathfrak{E})+_{F} \psi
$$

где $\psi \in \operatorname{ker}\left[\pi_{0}^{n}\right]_{F}$. Откуда мы получаем, что все эти элементы лежат в $F(\mathfrak{M})$, а значит, являются алгебраическими над $L$. Значит, поле $L\left(E_{F}(A \mathfrak{E})+{ }_{F} \psi\right)$ полно и содержит элемент

$$
\left(E_{F}(A \mathfrak{E})+{ }_{F} \psi\right)-{ }_{F} \psi=E_{F}(A \mathfrak{E}) .
$$


То есть

$$
L\left(E_{F}(A \mathfrak{E})+{ }_{F} \psi\right)=L\left(E_{F}(A \mathfrak{E})\right) .
$$

Приступим к доказательству п. 2.

Различные $A$, удовлетворяющие уравнению $A^{\varphi}-A=\alpha$, отличаются друг от друга на элемент из кольца $O_{0}$. Поэтому если $H_{\mathfrak{Z}}(\alpha)$ задается элементом $A$, а $H_{\mathfrak{Z}}(\alpha)^{\prime}$ - элементом $A^{\prime}$, причем $A^{\prime}=A+a$, где $a \in O_{0}$, то

$$
\begin{aligned}
H_{\mathfrak{Z}}(\alpha)^{\prime}-{ }_{F} H_{\mathfrak{Z}}(\alpha) & =E_{F}\left(\pi_{0}^{n} A^{\prime} \mathfrak{E}\right)-{ }_{F} E_{F}\left(\pi_{0}^{n} A^{\prime} \mathfrak{E}\right)=E_{F}\left(\pi_{0}^{n}\left(A^{\prime}-A\right) \mathfrak{E}\right) \\
& =E_{F}\left(\pi_{0}^{n} a \mathfrak{E}\right)=[a]_{F}\left(\left[\pi_{0}^{n}\right]_{F}\left(E_{F}(\mathfrak{E})\right)\right)=[a]_{F}\left(\left[\pi_{0}^{n}\right]_{F}(\mathfrak{Z})\right)=0 .
\end{aligned}
$$

Итак, $H_{\mathfrak{Z}}(\alpha)^{\prime}=H_{\mathfrak{Z}}(\alpha)$.

\section{2. Применение теории Хонды для формальных модулей}

2.1. Теория Хонды для формальных $\boldsymbol{O}_{0}$-модульных групп. Пусть $N / K_{0}-$ неразветвленное расширение локального поля $K_{0}, \pi_{0}$ - простой элемент в $K_{0}, \varphi$ - автоморфизм Фробениуса в $N / K_{0}$ :

$$
\varphi(a) \equiv a^{q} \bmod \pi_{0}
$$

для всех $a \in O_{N}$, где $q$ - число элементов поля вычетов $K_{0}$ поля $K_{0}$.

Пусть $O_{N} \llbracket \Delta \rrbracket^{\prime}$ - некоммутативное кольцо Робба от переменной $\Delta$, которое совпадает с $O_{N} \llbracket \Delta \rrbracket$ как левый $O_{N}$-модуль и имеет место соотношение

$$
\Delta a=\varphi(a) \Delta
$$

для любого $a \in O_{N}$.

Зададим действие оператора $\Delta$ на рядах из $O_{N}((X))$ :

$$
\Delta\left(\sum a_{i} X^{i}\right)=\sum \varphi\left(a_{i}\right) X^{q i}
$$

TeOpema 2 (см. [3], [5], [6]). 1) Пусть $\Lambda(\Delta)=1+c_{1} \Delta+\cdots \in N \llbracket \Delta \rrbracket$. Тогда ряд $\lambda(X)=$ $\Lambda(\Delta)(X)$ является логарифмом $\pi_{0}$-типической формальной $O_{0}$-модульной группы $F$ над $O_{N}$ тогда и только тогда, когда

$$
\Lambda=\pi_{0} U^{-1}
$$

для некоторого ряда $U=\pi_{0}+\alpha_{1} \Delta+\alpha_{2} \Delta^{2}+\cdots$ из кольиа Робба $O_{N} \llbracket \Delta \rrbracket^{\prime}$.

2) Для любой формальной $O_{0}$-модульной группь над $O_{N}$ с логарифмом $\lambda_{F}(X)$ существуют ряд $h(X)=X+\cdots \in O_{N} \llbracket X \rrbracket$ и ряд $U=\pi_{0}+\alpha \Delta+\cdots$ из кольца $O_{N} \llbracket \Delta \rrbracket^{\prime}$ такие, что

$$
\lambda_{F}(X)=\pi_{0} U^{-1}(h(X)) .
$$

3) Рядъ $\Lambda$ и $\Lambda^{\prime}$ соответствуют строго изоморфным формальным $O_{0}$-модульным группам над $O_{N}$ тогда и только тогда, когда

$$
U^{\prime}=\varepsilon U,
$$

əде $\varepsilon=1+c_{1} \Delta+\cdots \in O_{N} \llbracket \Delta \rrbracket^{\prime}$.

4) Ряд $f(X) \equiv a X \bmod \operatorname{deg} 2$ задает гомоморфизм формальных $O_{0}$-модульных групп $F u$ $F^{\prime}$ над $O_{N}$ тогда и только тогда, когда существует $c \in O_{N} \llbracket \Delta \rrbracket^{\prime}$ такой, что

$$
C U=U^{\prime} a .
$$

5) Пусть рядъ $\Lambda$ и $\Lambda^{\prime}$ из $N \llbracket \Delta \rrbracket$ удовлетворяют сравнению $\Lambda \equiv \Lambda^{\prime} \bmod \pi_{0}$. Тогда $\Lambda^{\prime}$ задает логарифм формалъной $O_{0}$-модулъной группы $F^{\prime}$ над $O_{N}$ тогда и толъко тогда, когда ряд 
$\Lambda$ задает логарифм некоторой формалъной $O_{0}$-модульной группъ $F$ над $O_{N}$. При этом $F^{\prime}$ строго изоморфна $F$.

6) В каждом классе изоморфных формальных $O_{0}$-модульных групп формальной $O_{0}$-высоть $h_{0}$ имеется канонический представитель $F_{\text {сап }}$ с логарифмом

$$
\lambda_{\text {can }}(X)=\left(1-\frac{\mathcal{A}(\Delta)}{\pi_{0}}(X)\right),
$$

где $\mathcal{A}(\Delta)=\alpha_{1} \Delta+\cdots+a_{h_{0}} \Delta^{h_{0}}$, при этом $\alpha_{1}, \ldots, \alpha_{h_{0}-1} \equiv 0 \bmod \pi_{0}, \alpha_{h_{0}} \in O_{0}^{*}$

2.2. Теорема о представлении логарифма формальной $O_{0}$-модульной группы. Пусть $K$ - конечное расширение $K_{0}, N$ - подполе инерции в $K / K_{0}$ и $\sigma$ - автоморфизм $Ф$ робениуса в $N / K_{0}$ :

$$
\sigma(\alpha) \equiv \alpha^{q} \quad \bmod \pi_{0} O_{N}, \quad \alpha \in O_{N}
$$

где $q=\# \overline{K_{0}}$.

Пусть $\Delta$ - оператор на кольце $O_{N}((X))$ :

$$
\Delta\left(\sum a_{i} x^{i}\right)=\sum \sigma\left(a_{i}\right) x^{q i}
$$

$W=O_{N} \llbracket \Delta \rrbracket^{\prime}-$ некоммутативное кольцо Робба с оператором $\Delta a=\sigma(a) \Delta, W^{\prime}=N \llbracket \Delta \rrbracket^{\prime}-$ аналогичное кольцо.

Если $f \in K \llbracket \Delta \rrbracket, g \in N \llbracket \Delta \rrbracket$, то

$$
f(\Delta) g(\Delta)=\sum c_{i} \Delta^{i}\left(a_{j} \Delta^{j}\right)=\sum c_{i} \sigma^{i}\left(a_{j}\right) \Delta^{i+j} .
$$

Пусть $t$ - переменная и $\sigma(t)=t^{q}$. Обозначим $\mathfrak{A}=O_{N} \llbracket t \rrbracket$, и пусть $\mathfrak{A} \llbracket \Delta \rrbracket^{\prime}-$ кольцо Робба, как В п. 2.1 .

Пусть $F(X, Y)$ - формальная $O_{0}$-модульная группа над $O$ с логарифмом $\lambda(X)=X+$ $c_{1} X^{q}+c_{2} X^{q^{2}}+\cdots, c_{i} \in K$, который запишем в виде

$$
\lambda(X)=\Lambda(\Delta)(X)
$$

где $\Lambda(\Delta)=1+c_{1} \Delta+c_{2} \Delta^{2}+\cdots \in K \llbracket \Delta \rrbracket$.

Теорема 3. Ряд $\Lambda(\Delta)$ можно представить в виде

$$
\Lambda(\Delta)=v(\Delta) u(\Delta)^{-1},
$$

¿əe

$$
\begin{aligned}
& v(\Delta)=\pi_{0}+c_{1} \Delta+c_{2} \Delta^{2}+\cdots \in K \llbracket \Delta \rrbracket, \\
& u(\Delta)=\pi_{0}+a_{1} \Delta+a_{2} \Delta^{2}+\cdots+a_{h_{0}} \Delta^{h_{0}}+\cdots \in W=O_{N} \llbracket \Delta \rrbracket^{\prime},
\end{aligned}
$$

при этом $a_{1}, \ldots a_{h_{0}-1} \vdots \pi_{0}, a_{h_{0}} \in O_{N}^{*}, h_{0}$ - формалъная $O_{0}$-высота $F$. Кроме того, $\pi_{0}^{l} \pi^{q} v(\Delta) \in$ $O \llbracket \Delta \rrbracket, l=\left[\log _{q} e /(q-1)\right]$.

ДокАЗАТЕльСтво фактически повторяет доказательство предложения 1.4.1 из работы [6].

2.3. Функция Артина-Хассе формальной $\boldsymbol{O}_{0}$-модульной группы. Пусть $K$ - конечное расширение поля $K_{0}, N$ - подполе инерции в $K / K_{0}, \varphi$ - автоморфизм Фробениуса в $N / K_{0}$ и $F(X, Y)$ - формальная $O_{0}$-модульная группа над $O=O_{K}$ формальной $O_{0}$-высоты $h$. Пусть

$$
\begin{aligned}
g: O_{N} \llbracket t \rrbracket & \rightarrow O, \\
t & \mapsto \pi,
\end{aligned}
$$


- кольцевой гомоморфизм (здесь $\pi$ - простой элемент в $K$ ). Тогда из свойств универсального $O_{0}$-формального закона [3] следует, что найдется формальный $O_{0} \llbracket t \rrbracket$-модульный закон $F_{t}(X, Y)$ такой, что

$$
F(X, Y)=g_{*}\left(F_{t}(X, Y)\right),
$$

где $g_{*}$ означает применение гомоморфизма $g$ к коэффициентам. При этом логарифм

$$
\begin{gathered}
\lambda_{F}(X)=g_{*}\left(\lambda_{F_{t}}(X)\right), \\
{[a]_{F}(X)=g_{*}\left([a]_{F_{t}}(X)\right)}
\end{gathered}
$$

для любого элемента $a \in O_{0}$.

Для формальной $O_{0} \llbracket t \rrbracket$-модульной группы $F_{t}(X, Y)$ согласно теореме 2 из п. 2.1 найдутся ряд $h(X) \in X+X O_{N} \llbracket t \rrbracket \llbracket X \rrbracket$ и ряд $U(\Delta)=\pi_{0}+O_{N} \llbracket t \rrbracket \llbracket \Delta \rrbracket \Delta$ такие, что

$$
\lambda_{F_{t}}(X)=\pi_{0} U(\Delta)^{-1} h(X) .
$$

Пусть $N^{\prime}$ - неразветвленное расширение поля $N, O_{N^{\prime}}-$ кольцо целых. Пусть $f(X) \in$ $O_{N^{\prime}} \llbracket X \rrbracket \llbracket t \rrbracket X$ и

$$
\Delta f=\Delta\left(\sum a_{i j} X^{i} t^{j}\right)=\sum \varphi\left(a_{i}\right) X^{q i} t^{q j} .
$$

ОпРЕДЕЛЕНиЕ 5. Зададим функцию Артина-Хассе $E_{F_{t}}(f)$ и обратную к ней $l_{F_{t}}(f)$ следующим образом:

$$
\begin{aligned}
E_{F_{t}}(f) & =\lambda_{F_{t}}^{-1}\left(\pi_{0} U(\Delta)^{-1} f\right), \\
l_{F_{t}}(f) & =\pi_{0}^{-1} U(\Delta) \lambda_{F_{t}}(f) .
\end{aligned}
$$

Проверим основные свойства функций $E_{F_{t}}$ и $l_{F_{t}}$.

ПрЕДЛОЖЕНИЕ 2. Имеем:

1) $E_{F_{t}}(f), l_{F_{t}}(f) \in O_{N^{\prime}} \llbracket t \rrbracket \llbracket X \rrbracket$;

2) $E_{F_{t}}\left(l_{F_{t}}(f)\right)=l_{F_{t}}\left(E_{F_{t}}(f)\right)=f$;

3) $E_{F_{t}}\left(f_{1}+f_{2}\right)=E_{F_{t}}\left(f_{1}\right)+{ }_{F_{t}} E_{F_{t}}\left(f_{2}\right), E_{F_{t}}(a f)=[a]_{F_{t}}\left(E_{F_{t}}(f)\right), a \in O_{0}$;

4) $l_{F_{t}}\left(f_{1}+F_{t} f_{2}\right)=l_{F_{t}}\left(f_{1}\right)+l_{F_{t}}\left(f_{2}\right), l_{F_{t}}\left([a]_{F_{t}} f\right)=a l_{F_{t}}(f), a \in O_{0}$.

ДокАзАтЕльство. Проверим сначала свойства 3) и 4). Из определения функций получаем

$$
\begin{aligned}
E_{F_{t}}\left(f_{1}+f_{2}\right) & =\lambda_{F_{t}}^{-1}\left(\pi_{0} U(\Delta)^{-1}\left(f_{1}+f_{2}\right)\right) \\
& =\lambda_{F_{t}}^{-1}\left(\pi_{0} U(\Delta)^{-1} f_{1}\right)+_{F_{t}} \lambda_{F_{t}}^{-1}\left(\pi_{0} U(\Delta)^{-1} f_{2}\right)=E_{F_{t}}\left(f_{1}\right)+_{F_{t}} E_{F_{t}}\left(f_{2}\right) .
\end{aligned}
$$

Аналогично из определения проверяется вторая часть свойства 3) и свойство 4).

Непосредственно из определения следует свойство 2).

Осталось проверить свойство 1). Если $f=\theta X^{m} t^{n}$, где $\theta$ - представитель Тейхмюллера в $O_{N^{\prime}}$, тогда $\Delta f=\left(\theta X^{m} t^{n}\right)^{q}=f^{q}$, и свойство 1) для $f$ вытекает из свойства 1$)$ для $X$. Заметим, что ряд $\lambda_{F_{t_{q}}}=\pi_{0} U(\Delta)^{-1} X$ является логарифмом формальной группы, изоморфной $F_{t}$. Теперь из определения $E_{F_{t}}$ ясно, что $E_{F_{t}}(X) \in O_{N^{\prime}}$ равносильно тому, что $E_{F_{t_{q}}}(X) \in$ $O_{N^{\prime}}$. Но $E_{F_{t_{q}}}(X)=X$, поэтому в данном случае свойство 1$)$ проверено.

Если $f=\pi_{0}^{k} \theta X^{m}$, то свойство 1 ) следует из свойства 3$)$.

Если $f=\alpha X^{m}$, где $\alpha=\sum \pi_{0}^{k} \theta_{k}$, то

$$
E_{F_{t}}\left(\alpha X^{m}\right)=\sum F_{t}\left[\pi_{0}^{k}\right]_{F_{t}}\left(E_{F_{t}}\left(\theta_{k} X^{m}\right)\right) \in O_{N^{\prime}}
$$

Если $f=\sum_{m \geqslant 1} \sum_{i \geqslant 0} \alpha_{m i} t^{i} X^{m}$, то свойство 1) следует из свойства 3$)$. 
Для проверки свойства 1) функции $l_{F_{t}}$ достаточно заметить, что $E_{F_{t}}$ сюръективно. Действительно, аналогично уже проведенным шагам можно увидеть, что

$$
E_{F_{t}}\left(\sum_{m \geqslant S} \sum_{i \geqslant 0} \alpha_{m i} t^{i} X^{m}\right)=\sum_{i \geqslant 0} \alpha_{S i} t^{i} X^{S}+\cdots,
$$

т.е. коэффициент при наименьшей степени $X$ сохраняется.

Итак, мы получили, что в образе $E_{F_{t}}$ есть набор рядов со всеми возможными минимальными степенями $X$ и коэффициентами при них. Тогда, очевидно, из них мы можем получить операцией $+F_{t}$ любой ряд из $O_{N^{\prime}} \llbracket X \rrbracket \llbracket t \rrbracket X$.

2.4. Примарные элементы для формальных $O_{0}$-модульных групп. В п. 1 были построены примарные элементы, в определение которых входит элемент $A$ из пополнения максимального неразветвленного расширения поля $N$. Для использования примарных элементов в явной конструкции теории полей классов и законов взаимности требуются примарные элементы, в определение которых входят только элементы исходного поля. Построение таких примарных элементов и является целью настоящего пункта.

Пусть $K$ - конечное расширение поля $K_{0}$ с кольцом целых $O=O_{K}$ и $F(X, Y)$ - формальная $O_{0}$-модульная группа над $O$. Пусть, как и ранее, $F_{t}$ - соответствующий группе $F$ формальный $O_{0}$-модульный закон, полученный с помощью гомоморфизма $g$ из п. 2.3.

Пусть $L$ - расширение поля $K$, содержащее все корни изогении $\left[\pi_{0}^{n}\right]_{F}(X)$ и $\mathfrak{Z}-$ один из первообразных корней: $\left[\pi_{0}^{n}\right]_{F}(\mathfrak{Z})=0,\left[\pi_{0}^{n-1}\right]_{F}(\mathfrak{Z}) \neq 0$.

Пусть $T$ - подполе инерции в $L / K_{0}, N \subset T$ и $\Pi$ - простой элемент в $L$; тогда разложению $\mathfrak{Z}:=\sum_{i \geqslant 1} \alpha_{i} \Pi^{i}, \alpha_{i} \in O_{T}$, соответствует ряд $\mathfrak{Z}(X)=\sum_{i \geqslant 1} \alpha_{i} X^{i} \in O_{T} \llbracket X \rrbracket$. Таким образом, определен ряд $l_{F_{t}}(\mathfrak{Z}(X)) \in O_{T} \llbracket t \rrbracket \llbracket X$.

Пусть $\varphi$ - автоморфизм Фробениуса в пополнении $\widetilde{T}$ максимального неразветвленного расширения поля $T$, т.е.

$$
\varphi(\alpha) \equiv \alpha^{q} \quad \bmod \pi_{0}, \quad \alpha \in O_{\widetilde{T}},
$$

где $q=\# K_{0}$. Тогда для любого $a \in O_{T}$ найдется элемент $A$ из $O_{\widetilde{T}}$, удовлетворяющий условию

$$
A^{\varphi}-A=a .
$$

Определим сначала $\pi_{0}^{n}$-примарный элемент для формальной $O_{0}$-модульной группы $F_{t}$ :

$$
H_{t}(a)=E_{F_{t}}\left(\pi_{0}^{n} A l_{F_{t}}(\mathfrak{Z}(X))\right) .
$$

Для формальной $O_{0}$-модульной группы $F$ определим $\pi_{0}^{n}$-примарный элемент следующим образом:

$$
H_{F}(a)=\left.H_{t}(a)\right|_{\substack{t=\pi=\pi \\ X=\Pi}}
$$

Пусть $\mathfrak{M}=\mathfrak{M}_{L}-$ максимальный идеал кольца целых поля $L$ и $F(\mathfrak{M})-$ формальный модуль, натянутый на $\mathfrak{M}$.

Пусть, как и раньше, $\sigma$ - образующий элемент группы Галуа расширения $T(A) / T$; тогда

$$
A^{\sigma}-A=\varkappa \in O_{0}
$$

Продолжение $\sigma$ на $\widetilde{T}$ обозначим той же буквой $\sigma$.

ТЕОрема 4. 1) Элемент $H_{F}(a)$ является $\pi_{0}^{n}$-примарным в модуле $F(\mathfrak{M})$, m.е. $H_{F}(a) \in L$ и $L\left(\frac{1}{\pi_{0}^{n}} H_{F}(a)\right) / L$ - конечное абелево неразветвленное расширение.

2) Примарный элемент $H_{F}(a)$ не зависит от выбора элемента $A$. 
ДокАЗАТЕЛЬСтво проводится аналогично доказательству теоремы 1 (приведем лишь набросок).

С помощью действия автоморфизма $\sigma$ мы убеждаемся в том, что коэффициенты ряда $H_{t}(a)$ лежат в поле $L$.

Затем мы замечаем, что

$$
\frac{1}{\left[\pi_{0}^{n}\right]} H_{F}(a)=\left.E_{F_{t}}\left(A l_{F_{t}}(\mathfrak{Z}(X))\right)\right|_{\substack{t=\pi \\ X=\Pi}}
$$

лежит в поле $\widetilde{L(A)}$, которое, в свою очередь, является неразветвленным над $L$ (так как $\widetilde{T(A)}$ неразветвленное над $T)$.

Далее, наша задача - получить примарный элемент, в определение которого входят только элементы исходного поля.

Итак, пусть $H_{t}(a)-\pi_{0}^{n}$-примарный элемент группы $F_{t}$. Логарифм $\lambda_{F_{t}}$ группы $F_{t}$ задается рядом $U(\Delta)=\pi_{0}-A(\Delta)$, где $\mathcal{A}(\Delta)=\alpha_{1} \Delta+\alpha_{2} \Delta^{2}+\cdots, \alpha_{i} \in O_{N} \llbracket X \rrbracket$.

Лемма 2. Имеет место равенство

$$
\frac{1}{\pi_{0}^{n}} H_{t}(a)=E_{F_{t}}\left(A l_{F_{t}}(\mathfrak{Z}(X))=E_{F_{t}}\left(\sum_{i \geqslant 1} \frac{\alpha_{i} a_{i} \Delta^{i}}{\pi_{0}} \lambda_{F_{t}}(\mathfrak{Z}(X))\right)+{ }_{F_{t}} \lambda_{F_{t}}^{-1}\left(A \lambda_{F_{t}}(\mathfrak{Z}(X))\right),\right.
$$

где $a_{i}=a+a^{\varphi}+\cdots+a^{\varphi^{i-1}}, \varphi$ - автоморбизм Фробениуса в $N / K_{0}$.

ДокАзАТЕЛьство. Из определения функции $E_{F_{t}}$ следует, что

$$
E_{F_{t}}\left(\left(1-\frac{\mathcal{A}(\Delta)}{\pi_{0}}\right) A \lambda_{F_{t}}(\mathfrak{Z}(X))\right)=\lambda_{F_{t}}^{-1}\left(A \lambda_{F_{t}}(\mathfrak{Z}(X))\right) \text {. }
$$

Отсюда получаем

$$
\begin{aligned}
E_{F_{t}}\left(A l_{F_{t}}(\mathfrak{Z}(X))\right)= & E_{F_{t}}\left(A\left(1-\frac{\mathcal{A}(\Delta)}{\pi_{0}}\right) \lambda_{F_{t}}(\mathfrak{Z}(X))\right) \\
& \quad+{ }_{F_{t}} \lambda_{F_{t}}^{-1}\left(A l_{F_{t}}(\mathfrak{Z}(X))\right)-{ }_{F_{t}} E_{F_{t}}\left(\left(1-\frac{\mathcal{A}(\Delta)}{\pi_{0}}\right) A \lambda_{F_{t}}(\mathfrak{Z}(X))\right) \\
= & E_{F_{t}}\left(\sum \frac{\alpha_{i}\left(A^{\varphi^{i}}-A\right)}{\pi_{0}} \lambda_{F_{t}}(\mathfrak{Z}(X))\right)+{ }_{F_{t}} \lambda_{F_{t}}^{-1}\left(A \lambda_{F_{t}}(\mathfrak{Z}(X))\right) .
\end{aligned}
$$

Осталось заметить, что из равенства $A^{\varphi}-A=a$ следует, что $A^{\varphi^{i}}-A=a_{i}$.

Пусть теперь

$$
P_{F}(a)=\left.E_{F_{t}}\left(\pi_{0}^{n-1}\left(\sum_{i \geqslant 1} \alpha_{i} a_{i} \Delta^{i}\right) \lambda_{F_{t}}(\mathfrak{Z}(X))\right)\right|_{\substack{t=\pi \\ X=\Pi}}
$$

Тогда имеет место следующая теорема.

Теорема 5. Выполнено $H_{F}(a)=P_{F}(a)$. И, тем самым, мы дали определение примарного элемента, под которое подходят только элементы исходного поля.

ДокАЗАТЕЛЬСтво. Согласно лемме нам надо проверить только следующее равенство:

$$
\left.\lambda_{F_{t}}^{-1}\left(\pi_{0}^{n} A \lambda_{F_{t}}(\mathfrak{Z}(X))\right)\right|_{\substack{t=\pi \\ X=\Pi}}=0 .
$$

После подстановки получим необходимое равенство

$$
\lambda_{F}\left(\pi_{0}^{n} A \lambda_{F}(\mathfrak{Z})\right)=0,
$$

которое в свою очередь следует из того, что $\mathfrak{Z}$ как корень изогении $\left[\pi_{o}^{n}\right]_{F}$ является также корнем логарифма. 


\section{Список литературы}

[1] С. В. Востоков, "Явная форма закона взаимности", Изв. АН СССР. Сер. матем., 42:6 (1978), $1288-1321$.

[2] С. В. Востоков, "Норменное спаривание в формальных модулях", Изв. АН СССР. Сер. матем., 43:4 (1979), 765-794.

[3] M. Hazewinkel, Formal Groups and Applications, Pure Appl. Math., 78, Academic Press, New York, 1978.

[4] E. Artin, H. Hasse, "Die beiden Ergänzungssätze zum Reziprozitatsgesetz der $l^{n}$-ten Potenzreste im Körper der $l^{n}$-ten Einheitswurzeln", Abh. Math. Semin. Univ. Hamb., 6 (1928), 146-162.

[5] T. Honda, "On the theory of commutative formal groups", J. Math. Soc. Japan, 22:2 (1970), 213-246.

[6] М.В. Бондарко, С. В. Востоков, "Явная классификация формальных групп над локальными полями”, Теория чисел, алгебра и алгебраическая геометрия, Сборник статей. К 80-летию со дня рождения академика Игоря Ростиславовича Шафаревича, Тр. МИАН, 241, Наука, М., 2003, 43-67. 